\begin{tabular}{|c|c|c|}
\hline & $\begin{array}{c}\text { International Journal of Current Research in } \\
\text { Biosciences and Plant Biology }\end{array}$ \\
\hline EXCELLENT \\
PUBLISHERS
\end{tabular}

\title{
Molecular Cloning and Characterization Analysis of a Gene Encoding Cup-shaped Cotyledon (CUC1) from Phoebe neurantha (Hemsl.) Gamble
}

\author{
Pengyu Yu, Tingting Tao, Yuemiao Wu, Yue Fei and Bo Xiao*
}

Phoebe Germplasm Resources Evaluation and Innovation Center of Yangtze University, Jingzhou 434025, China

*Corresponding author.

\begin{tabular}{|c|c|}
\hline Abstract & Article Info \\
\hline $\begin{array}{l}\text { Cup-shaped cotyledon }(C U C) \text { gene is a kind of border-specific gene, which is leaf edge } \\
\text { cracking and complex leaflet development. CUC plays an important role in shoot apical } \\
\text { meristem formation and leaf margin development in Phoebe neurantha (Hemsl.) Gamble. }\end{array}$ & $\begin{array}{l}\text { Accepted: } 18 \text { December } 2016 \\
\text { Available Online: 06 January } 2017\end{array}$ \\
\hline $\begin{array}{l}\text { In this paper, a } C U C \text { gene designated as } P n C U C l \text { was successfully cloned from } P \text {. } \\
\text { neurantha (Hemsl.) Gamble. The full-length of } P n C U C 1 \text { was } 1317 \text {-bp and contained a }\end{array}$ & Keywords \\
\hline $\begin{array}{l}\text { 1008-bp open reading frame. It encoded a } 335 \text {-amino-acid protein with a calculated } \\
\text { molecular weight of about } 37.8 \mathrm{kDa} \text { and isoelectric point of } 7.04 \text {. Sequence comparison } \\
\text { revealed PnCUC1 showed extensive homology with CUCs from other plant species. } \\
\text { Phylogenetic tree analysis revealed that PnCUC1 were high similar to previously } \\
\text { described CUC1 proteins from other plant species and shared the same ancestor in } \\
\text { evolution with other CUCs, indicating that PnCUC1 belonged to a multi-gene family. The } \\
\text { cloning and characterization of PnCUC1 gene will be helpful to further study the role of } \\
P n C U C 1 \text { gene in the regulation of leaf margin and braches development in P. neurantha } \\
\text { (Hemsl.) Gamble. }\end{array}$ & $\begin{array}{l}\text { Leaf development } \\
\text { Phoebe neurantha (Hemsl.) Gamble } \\
\text { PnCUC1 }\end{array}$ \\
\hline
\end{tabular}

\section{Introduction}

Phoebe neurantha (Hemsl.) Gamble is a evergreen tree species of Phoebe Nees, Lauraceae. Due to its straight trunk and beautiful tree shape, P. neurantha (Hemsl.) Gamble is a good landscape tree and widely used in the garden, which is usually considered as plant landscaping tree and landscape background tree. $P$. neurantha (Hemsl.) Gamble is also a kind of precious timber trees resource of Chinese traditional timber trees, because of its unique fragrance and fine texture (Hai-Shan et al., 2014).

Leaf development and mechanism of leaf morphogenesis are hot topics of plant developmental biology. With the aid of the model plant mutants research, many key genes of leaf development has been cloned and identified (Nicotra et al., 2011). However, the regulatory mechanism of leaf development in other plant remains to be illuminated. During pattern formation in plants and animals, groups of cells are divided into domains that acquire different developmental fates. This process requires the establishment of precise gene expression patterns that are maintained despite continuous growth and cell division. A recently discovered class of small RNAs, the microRNAs (miRNAs) involved in gene expression regulation may contribute to this (Laufs 
et al., 2004). MiRNAs are small, single-stranded RNAs molecules of about $21 \mathrm{bp}$ in length, that posttranscriptionally regulate gene expression (Kidner, 2010; Pulido and Laufs, 2010). The biological function of miRNAs has investigated in many plants, including Arabidopsis thaliana (Sunkar and Zhu, 2004), Oryza sativa (Sunkar et al., 2005), Zea mays (Mica et al., 2006), Triticum aestivum (Yao et al., 2007), Nicotiana tabacum (Billoud et al., 2005), Populus tomentosa (Lu et al., 2005) and Pteridophyta (Axtell and Bartel, 2005).

The cup-shaped cotyledon (CUC) genes CUC1, $C U C 2$, and $C U C 3$ are required to demarcate primordium boundaries as well as promote meristem formation (Takada et al., 1991). Recent studies have shown that miR164 plays the key role in the development of the leaf margin. The target gene of miR164 is the transcription factor of CUC1, CUC2, NAC1 of NAM / CUCc family (Mallory et al., 2004). $C U C 1$ and $C U C 2$, a kind of border-specific gene, plays an important role in leaf edge cracking and complex leaflet development. The balance between the miR164 and $C U C 2$ determines the depth of cleft lobes, the stronger $C U C 2$ activity, the deeper the lobes, the miR164 mutant and the phenotype of $C U C$ overexpression, Mutation occurs so that miR164 cannot mediate cleavage of the CUC mRNA, resulting in the accumulation of CUC mRNA, Resulting in Arabidopsis leaf margin to produce deep cleavage, while overexpression of miR164 Arabidopsis plant leaf margin cleft engraved disappearance (Nikovics et al., 2006). Expression of mutated miRl64 causes uncontrolled post-transcriptional regulation of the $C U C$ gene while driving $C U C 1$ expression with the CUC2 promoter, and the Arabidopsis single leaf becomes a compound leaf (Hasson et al., 2011). However, the enzymes involved in the leaf margin development in P. neurantha (Hemsl.) Gamble are not well understand. The identification of the $C U C$ genes and the genetic sequences are important for further studies of leaf development in P. neurantha (Hemsl.) Gamble. To date, little is known about the gene encoding $C U C$ in $P$. neurantha (Hemsl.) Gamble. Therefore, we cloned a new $C U C 1$ gene from $P$. neurantha (Hemsl.) Gamble and analysis the sequence of PnCUCl.

\section{Materials and methods}

Phoebe neurantha (Hemsl.) Gamble leaves were collected from botanical garden at Yangtze University, and immediately placed in a $-80^{\circ} \mathrm{C}$ freezer. Both the primers synthesis and DNA sequencing were performed by Shanghai Sangon Biotechnology Company, in China. Agarose Gel DNA purification Kit Ver.4.0, pMD18-T vector kit, AMV Reverse Transcriptase, dNTPs, RNase in and Taq DNA polymerase were purchased from Takara Company (Dalian, China).

\section{Cloning the full-length cDNA of PnCUC1}

Total RNA of $P$. neurantha (Hemsl.) Gamble seedlings was isolated using the TaKaRa MiniBEST Plant RNA Extraction kit (Dalian, China) according to the manufacturer's instructions. The concentration and quality of the RNA were measured via spectrophotometry and agarose gel electrophoresis. The primers PnCUC1-u (5'-ATGGCCCCAGCAGCTGTAGT-3') and PnCUC1-d (5'-TTTCGTTGTGGAACTTCTG-3') were designed and synthesized according to the gene annotation of $P$. neurantha (Hemsl.) Gamble in the transcriptome database. The full-length cDNA sequence of PnCUC1 was amplified with the one-step RT-PCR kit (Dalian TaKaRa, Dalian, China) under the following condition: $94^{\circ} \mathrm{C}$ for $3 \mathrm{~min} ; 33$ cycles of amplification at $94^{\circ} \mathrm{C}$ for $20 \mathrm{~s}, 56^{\circ} \mathrm{C}$ for $40 \mathrm{~s}$, and $72^{\circ} \mathrm{C}$ for $60 \mathrm{~s}$; and a final extension at $72^{\circ} \mathrm{C}$ for $7 \mathrm{~min}$. The PCR products were purified, ligated into pMD18-T vector, and introduced into $E$. coli strain DH5a followed by sequencing.

\section{Bioinformatics and molecular evolution analysis}

Sequence assembly was performed with programs of DNAstar (http://www.dnastar.com). The nucleotide sequence, deduced amino acid sequence and open reading frame (ORF) were analyzed, and the sequence comparison was conducted through database search (http://www.ncbi. nlm.nih.gov).

The calculated isoelectric point $(\mathrm{pI})$ and molecular weight of the PnCUC1 protein were computed with the software of Compute $\mathrm{pI} / \mathrm{Mw}$ Tool at http://web.expasy. org/compute_pi/. Multiple sequence alignment was performed with the software Vector NTI 11.5 program. Phylogenetic analysis of PnCUC1 and other CUC from other plants were aligned with CLUSTALX 2, and subsequently, a phylogenetic tree was constructed by the neighbor-joining (NJ) method with MEGA 6 software (Kumar et al., 2004). 


\section{Results}

\section{Cloning and characterization of the full-length cDNA of PnCUC1 gene}

A total of 49,561 unigenes have been identified in $P$. neurantha (Hemsl.) Gamble RNA-Seq database through annotation against public protein databases (data not published). Among these unigenes, one CUC member was identified based on the annotation information. Using a pair of specific primers based on the CUC unigene (Gene ID: c41381) of transcriptome data, we performed PCR using cDNA as the template, and a 1317-bp fragment was amplified. It contained a 1008-bp open reading frame (ORF) encoding a 335-amino-acid protein (Fig. 1). The nucleotide sequence of $\mathrm{PnCUC1}$ was $82,80,78,76$ and $74 \%$ identical to those of the CUC1 genes from Nelumbo nucifera, Theobroma cacao, Beta vulgaris, Eucalyptus grandis, and Glycine max, respectively (Table 1 ). The results indicate that the gene we cloned is a member of the $C U C$ gene family.

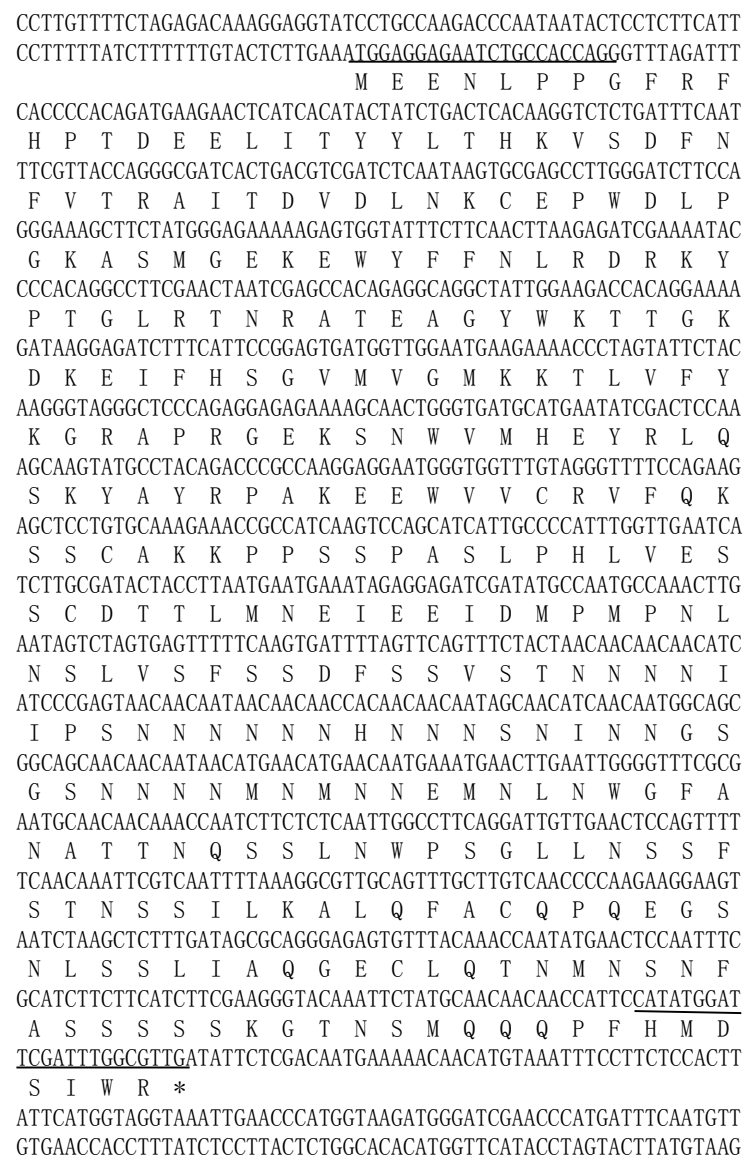

1261 ATCATGGTTGCAAAGCTTCCTAGATATTAGCTAGAAGACAACTATATATTCCCACG sequence of $P n C U C 1$. The primer sequences are underlined.
Table 1. Nucleotide sequence of PnCUC1 similarity to the $C U C$ genes of other plant species.

\begin{tabular}{llll}
\hline Species & Accession no. & E-value & $\begin{array}{l}\text { Homology } \\
(\boldsymbol{\%})\end{array}$ \\
\hline Nelumbo nucifera & XM_010265607.1 & $8 \mathrm{e}-106$ & 82 \\
Theobroma cacao & XM_007036468.2 & $4 \mathrm{e}-104$ & 80 \\
Beta vulgaris & XM_010680148.1 & $5 \mathrm{e}-73$ & 78 \\
Eucalyptus grandis & XM_010038590.1 & $2 \mathrm{e}-56$ & 76 \\
Glycine max & XM_003541118.3 & $3 \mathrm{e}-26$ & 74 \\
\hline
\end{tabular}

\section{Characterization of the deduced PnCUC1 protein}

By using the software of Compute $\mathrm{pI} / \mathrm{Mw}$ Tool, the calculated isoelectric point ( $\mathrm{pI}$ ) and molecular weight of the PnCUC1 were predicted to be 7.04 and $37.8 \mathrm{kDa}$, respectively. Sequence comparison by performing BlastP search showed that PnCUC1 exhibited high similarity to CUC proteins from other plants (Fig. 2). The deduced PnCUC1 protein sequence showed 77, 73, $73,70,69,61$ and $60 \%$ identities to the counterparts of Manihot esculenta, Nicotiana tabacum, Nicotiana sylvestris, Solanum tuberosum, Nicotiana tomentosiformis, Arachis ipaensis and Elaeis guineensis, thereby indicating that PnCUC1 belongs to plant CUC superfamily.

\section{Molecular evolution analysis}

To investigate the evolutionary relationships among PnCUC1 and CUC proteins from other plants, a phylogenetic tree was constructed by using software Clustal X2 and MEGA6 with the neighbor-joining (NJ) method. As showed in Fig. 3, the evolutionary tree was divided into five distinct categories. The results highlighted all plants derived from a common ancester in the evolution using CUC as outgroup, no matter whether they belonged to the Solanaceae, Leguminosae, Lauraceae, Rosaceae or Brassicaceae. PnCUC1 belonged to Lauraceae had a close relationship to other CUC proteins. These results suggest that PnCUC1 shares a common evolutionary with other plant CUC proteins based on conserved structure and sequence characteristics, such as amino acid homologies and conserved motifs, respectively.

\section{Discussion}

Leaf shape can significantly affect plant photosythesis, yield, and commercial characters and so on, its diversity is produced by alterations of the margin. Compared to complement leaf, a deeply labe leafed would more adapt 
the adversity. Leaf-edge cleavage could reduce the distance of heat transfer and have more effective in combating to heat damage to facilitate plant adaptation to high temperatures (Vogel, 2009). In addition, leafedge cleavage imparts a plasticity that extends longitudinally to the leaves and can respond more quickly to compete for limited light sources (Semchenko and Zobel, 2007). Leaf-edge cleavage also could increase the hydraulic efficiency to better adapt the drought conditions (Siso et al., 2001; Jiang et al., 2000).

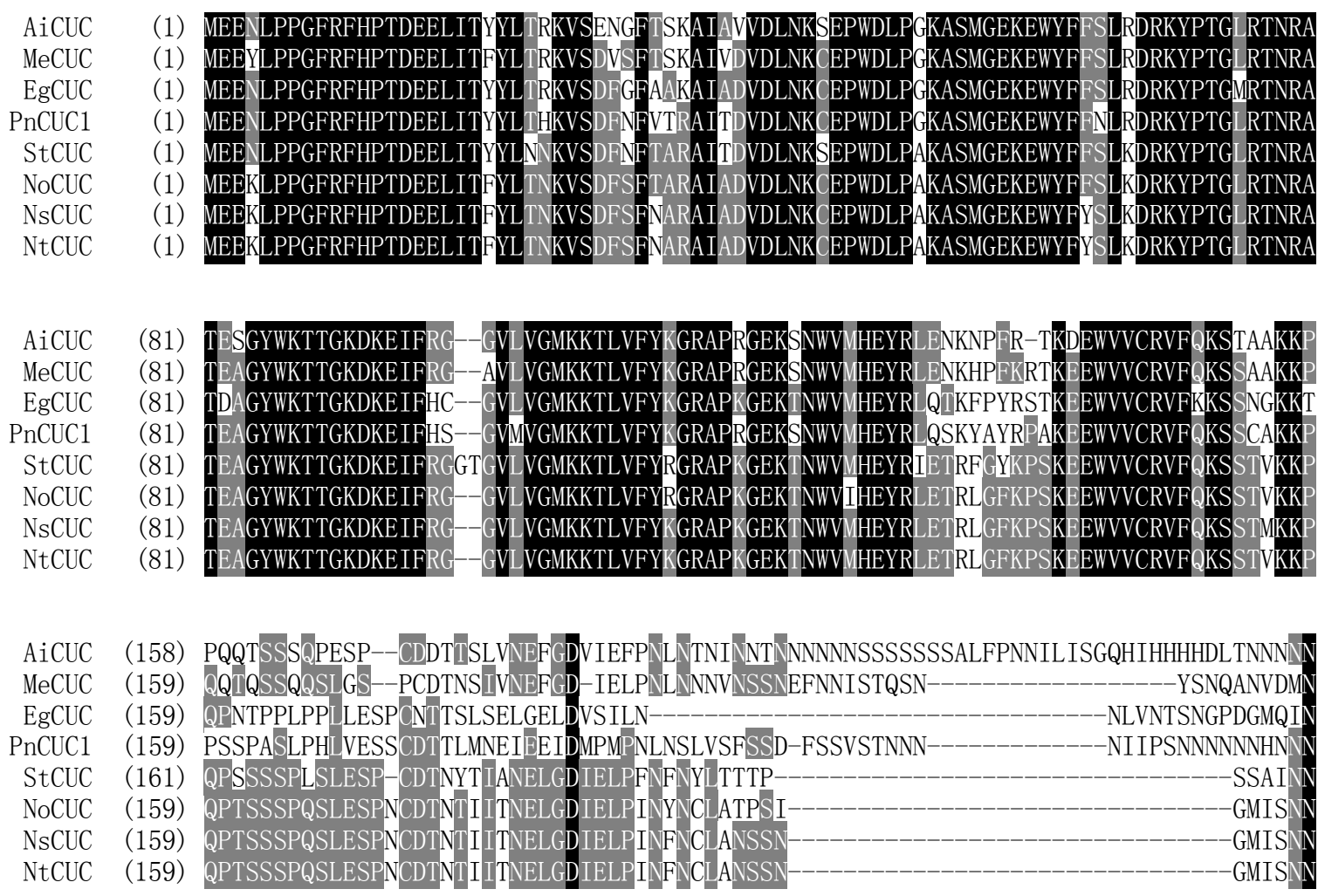

AiCUC (236) NNVNTNMNLAMNWPP----------SSDHNVPWPSVGLLNPSISSM SLILKALQLRNNYQREVASTFAPSSYIMPHH

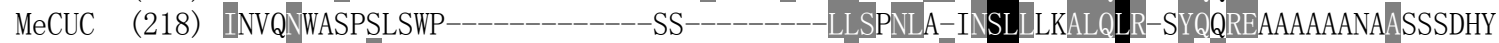

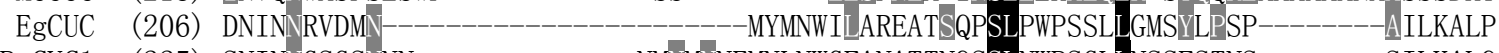

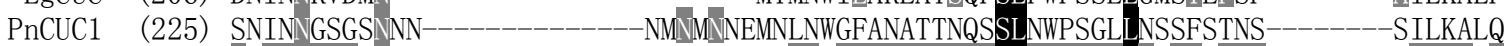

StCUC (207) ISLHNYNNDNINLAAAAAAATREAAAANTHPLLPWSSNLLSSNLSSVNSLLFRALQLR-GYSPREQA----TSSTTTTHD

NoCUC (207) ISLHNYNNENM------------NMNLNMNLASSLLNSSNLSSVNSLLFRALQL

NSCUC (207) ISLHNYNNE---------------NMNMNLASSLL NSSNLSS VNSLLFRALQL

NtCUC

(207)

NMNMNLASSLLNSSNLSSVNSLLFRALQL

AiCUC (305) QGVVVPHQQVI IGTNNDDLITTSSNLINASSSSSSKVLECMPHQQQQQEQPFNLDSL----

MeCUC (274) SFLTQGTSIPQFGTDLNSNVQGSS---_----SSSRVLETVQQPVQQ--EQPFILDSIW---

EgCUC (255) FNGYQPQEDANLTSLSSFAAQGNPMLGESRSSFPSSS-TKGTEVPQQQ--HPFDQEPIWRGY

PnCUC1 (283) FACQ-PQEGS---NLSSLIAQGECLQTNMNSNFASSSSSKGTNSMQQ---QPFHMDSIWR--

StCUC (282) YAFMLPQE-NILTTQFGNDFAVNSTG----APSSSMALDNSVQNQQPQ-EQLYKLDSNIW--

NoCUC (263) YSFMPPIQGNISQFANDNHFSSNFVANIGAIPSSSMVFDNSVQQQQTQ-EQPYKLDSNIW--

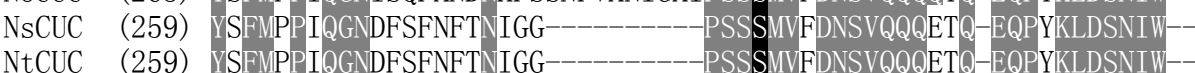

Fig. 2: The multiple alignments of PnCUC1 amino acid sequence with other CUC proteins. The completely identical amino acids are indicated with white foreground and black background. The conserved amino acids are indicated with white foreground and grey background. Non-similar amino acids are indicated with black foreground and white background. The species, protein names and GenBank accession numbers are as following: Elaeis guineensis: EgCUC (XP_010916375.1); Nicotiana tabacum: NtUC (XP_016433985.1); Nicotiana tomentosiformis: NoCUC (XP_009620409.1); Nicotiana sylvestris: NsCUC (XP_009779881.1); Manihot esculenta: MeCUC (ALC79053.1); Solanum tuberosum: StCUC (XP_006353806.2); Arachis ipaensis: AiCUC (XP_016203627.1). 


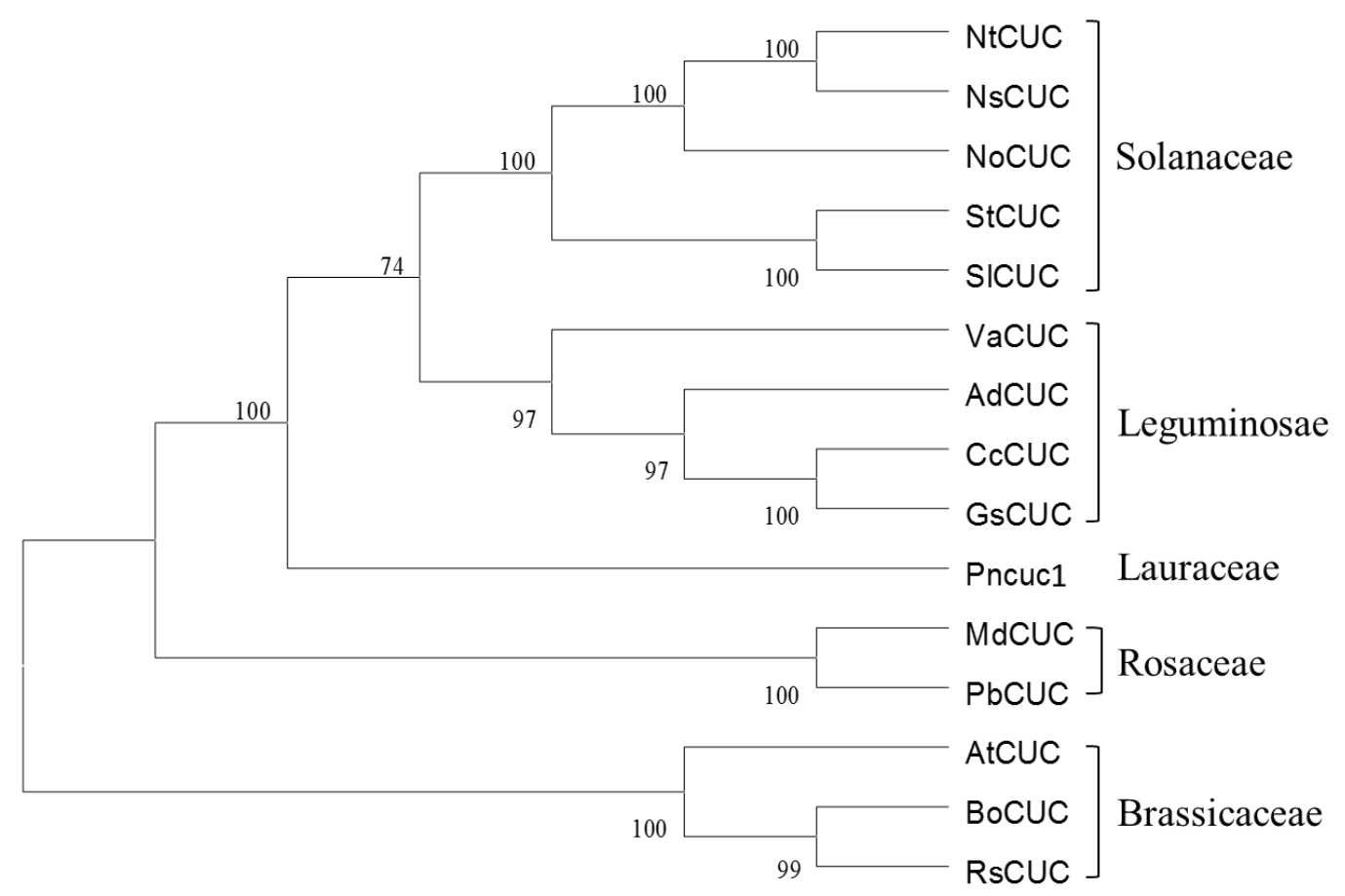

Fig. 3: Phylogenetic tree of CUC from different species using the Neighbor-joining method. The numbers of nodes represent the percentage of boot strap value obtained from 1000 sampling. Bar 0.01 shows the substitutions per nucleotide position. The species, protein names and GenBank accession number are as following: Cajanus cajan: CcCUC (KYP69700.1); Glycine soja: GsCUC (KHN20732.1); Vigna angularis: VaCUC (XP_017441934.1); Arachis duranensis: AdCUC (XP_015966676.1); Nicotiana tabacum: NtCUC (XP_016433985.1); Nicotiana tomentosiformis: NoCUC (XP_009620409.1); Nicotiana sylvestris: NsCUC (XP_009779881.1); Solanum tuberosum: StCUC (XP_006353806.2); Solanum lycopersicum: SICUC (XP_004252235.1); Malus domestica: MdCUC (XP_008355378.1); Pyrus $x$ bretschneideri: PbCUC (XP_009339299.1); Arabidopsis thaliana: AtCUC (AAP82630.1); Brassica oleracea: BoCUC (ADU76441.1); Raphanus sativus: RsCUC (ADU76440.1).

The microRNA miR164 family negatively regulates CUC1 and CUC2 expression levels: overexpression of miR164 in transgenic plants leads to a reduction in the mRNA levels of CUC1 and CUC2 (Kwon et al., 2006), whereas mutants that affect miR164 accumulation result in increased $C U C 1$ and $C U C 2$ expression, and in an enlarged boundary domain (Baker et al., 2005). Up until now, the genomic analysis of $C U C$ gene has only been reported in some model-plants, such as Arabidopsis thaliana (Bellesboix et al., 2006; Aida et al., 1997) and rice (Hu et al., 2006).

Mapping and cloning of leaf lobe genes will provide an insight into mechanism of leaf shape development and improving cultivar in P. neurantha (Hemsl.) Gamble. In this study, a 1317 bp full-length cDNA of the PnCUC1 gene was isolated from $P$. neurantha (Hemsl.) Gamble. The PnCUCl gene encodes a 335 amino acid protein. Our multiple alignments showed that the deduced PnCUC1 sequence exhibited high similarity to CUC proteins from other plants. The phylogenetic tree indicated that PnCUC1 has a distinct and ancient relationship with the CUCs from other plant species.

\section{Conclusion}

In conclusion, in this present investigation, we have successfully cloned and characterized the gene encoding cup-shaped cotyledon (CUC) involved in the regulation of leaf margin and braches development in P. neurantha (Hemsl.) Gamble. Multiple sequence alignment of the deduced PnCUC1 showed highest identity to other plants. Therefore, our study will be helpful to understand the mechanism of leaf shape development in P. neurantha (Hemsl.) Gamble. Further studies on the identification of genes related to leaf development of this plant would not only be useful for understanding the shoot apical meristem formation and leaf margin development but will also provide molecular wealth for cultivate new varieties of $P$. neurantha (Hemsl.) Gamble. 


\section{Conflict of interest statement}

Authors declare that they have no conflict of interest.

\section{Acknowledgement}

This work was supported by the Natural Science Foundation of Hubei Province (2013CFA039), and the Science and Technology Support Plan of Hubei Province (2013BBB24).

\section{References}

Aida, M., Ishida, T., Fukaki, H., Fujisawa, H., Tasaka, M., 1997. Genes involved in organ separation in Arabidopsis: An analysis of the cup-shaped cotyledon mutant. Plant Cell. 9(6), 841-57.

Axtell, M. J., Bartel, D. P., 2005. Antiquity of microRNAs and their targets in land plants. Plant Cell. 17(6), 1658-1673.

Baker, C. C., Sieber, P., Wellmer, F., Meyerowitz, E. M., 2005. The early extra petals 1 mutant uncovers a role for microRNA miR164c in regulating petal number in Arabidopsis. Curr. Biol. 15, 303-315.

Bellesboix, E., Hamant, O., Witiak, S. M., Morin, H., Traas, J., Pautot, V., 2006. Knat6: An Arabidopsis homeobox gene involved in meristem activity and organ separation. Plant Cell. 18(8), 1900-1907.

Billoud, B., Paepe, R. D., Baulcombe, D., Boccara, M., 2005. Identification of new small non-coding RNAs from tobacco and Arabidopsis. Biochim. 87(9-10), 905-910.

Hai-Shan, H. E., Jian, Q., Guo, M. L., Gan, C. T., Pan, Z. H., 2014. Investigation of wood species prone to forming zone lines. For. Res. 27(6), 776-780.

Hasson, A., Plessis, A., Blein, T., Adroher, B., Grigg, S., Tsiantis, M., 2011. Evolution and diverse roles of the cup-shaped cotyledon genes in Arabidopsis leaf development. Plant Cell. 23(1), 54-68.

Hu, H., Dai, M., Yao, J., Xiao, B., Li, X., Zhang, Q., Xiong, L., 2006. Overexpressing a NAM, ATAF, and CUC (NAC) transcription factor enhances drought resistance and salt tolerance in rice. Proc. Natl. Acad. Sci. (USA). 103(35), 12987-12992.

Jiang, C., Wright, R. J., Woo, S. S., Delmonte, T. A., Paterson, A. H., 2000. QTL analysis of leaf morphology in tetraploid Gossypium (cotton). Theor. Appl. Genet. 100(3), 409-418.

Kidner, C. A., 2010. The many roles of small RNAS in leaf development. J. Genet. Genom. 37(1), 1321.
Kumar, S., Tamura, K., Nei, M., 2004. MEGA3: Integrated software for molecular evolutionary genetics analysis and sequence alignment. Brief Bioinform. 5, 150-163.

Kwon, C. S., Hibara, K. I., Pfluger, J., Bezhani, S., Metha, H., Aida, M., Wagner, D., 2006. A role for chromatin remodeling in regulation of $C U C$ gene expression in the Arabidopsis cotyledon boundary. Development. 133(16), 3223-3230.

Laufs, P., Peaucelle, A., Morin, H., Traas, J., 2004. MicroRNA regulation of the $c u c$ genes is required for boundary size control in Arabidopsis meristems. Development, 131(17), 4311-22.

Lu, S., Sun, Y. H., Shi, R., Clark, C., Li, L.,Chiang, V. L., 2005. Novel and mechanical stress-responsive microRNAs in Populus trichocarpa that are absent from Arabidopsis. Plant Cell. 17(8), 21862203.

Mallory, A. C., Dugas, D.V., Bartel, D.P., Bartel, B., 2004. MicroRNA regulation of NAC-domain targets is required for proper formation and separation of adjacent embryonic, vegetative, and floral organs. Curr. Biol. 14(12), 1035-46.

Mica, E., Gianfranceschi, L., Pè, M. E., 2006. Characterization of five microRNA families in maize. J. Exp. Bot. 57(11), 2601-2612.

Nicotra, A. B., Leigh, A., Boyce, C. K., Jones, C. S., Niklas, K. J., Royer, D. L., Tsukaya H., 2011. The evolution and functional significance of leaf shape in the angiosperms. Funct. Plant Biol. 38(7), 535552.

Nikovics, K., Blein, T., Peaucelle, A., Ishida, T., Morin, H., Aida, M., Laufs, P., 2006. The balance between the mir164a and cuc2 genes controls leaf margin serration in Arabidopsis. Plant Cell. 18(11), 29292945.

Pulido, A., Laufs, P., 2010. Co-ordination of developmental processes by small RNAs during leaf development. J. Exp. Bot. 61(5), 1277-91.

Semchenko M, Zobel K., 2007. The role of leaf lobation in elongation responses to shade in the rosettefornting for Serratula tinctoria (Astcraccac). Ann. Bot-London. 100(1), 83-90.

Siso, S., Camarero, J., Gil-Pelegrin, E., 2001. Relationship between hydraulic resistance and leaf morphology in broadleaf Quercus species: A new interpretation of leaf lobation. Trees. 15(6), 341345.

Sunkar, R., Girke, T., Jain, P.K., Zhu, J., 2005. Cloning and characterization of microRNAs from rice. Plant Cell. 17(5), 1397-1411. 
Sunkar, R., Zhu, J. K., 2004. Novel and stress-regulated microRNAs and other small RNAs from Arabidopsis. Plant Cell. 16(8), 2001-2019.

Takada, S., Hibara, K., Ishida, T., Tasaka, M., 1991. The cup-shaped cotyledon1 gene of Arabidopsis regulates shoot apical meristem formation. Development. 128(7), 1127-1135.
Vogel, S., 2009. Leaves in the lowest and highest winds: Temperature, force and shape. New Phytol. 183(1), 13-26.

Yao, Y.Y., Guo, G. G., Ni, Z. Y., Sunkar, R., Du, J., Zhu, J. K., Sun, Q., 2007. Cloning and characterization of microRNAs from wheat (Triticum aestivum L.). Genome Biol. 8(6), 96-103.

\section{How to cite this article:}

Yu, P., Tao, T., Wu, Y., Fei, Y., Xiao, B., 2017. Molecular cloning and characterization analysis of a gene encoding cup-shaped cotyledon (CUC1) from Phoebe neurantha (Hemsl.) Gamble. Int. J. Curr. Res. Biosci. Plant Biol. 4(1), 612. doi: http://dx.doi.org/10.20546/ijcrbp.2017.401.002 